\title{
Differences in intestinal microbiome are associated with the mortality of COVID-19 patients in intensive care units
}

\author{
Dong $\mathrm{Wu}^{1,2 \dagger}$, Kaichen $\mathrm{Zhou}^{3 \dagger}$, Meng Xiao ${ }^{2,4 \dagger}$, Zhihua Liu ${ }^{3 *}$ \& Shuyang Zhang ${ }^{2,5^{*}}$ \\ ${ }^{1}$ Department of Gastroenterology, Peking Union Medical College Hospital, Chinese Academy of Medical Sciences \& Peking Union Medical \\ College, Beijing 100730, China; \\ ${ }^{2}$ National Key Laboratory of Complex Severe and Rare Diseases, Peking Union Medical College Hospital, Chinese Academy of Medical \\ Sciences \& Peking Union Medical College, Beijing 100730, China; \\ ${ }^{3}$ Institute for Immunology and School of Medicine, Tsinghua University, Beijing 100084, China; \\ ${ }^{4}$ Department of Clinical Laboratory, Peking Union Medical College Hospital, Chinese Academy of Medical Sciences \& Peking Union Medical \\ College, Beijing 100730, China; \\ ${ }^{5}$ Department of Cardiology, Peking Union Medical College Hospital, Chinese Academy of Medical Sciences \& Peking Union Medical College, \\ Beijing 100730, China
}

Received December 16, 2021; accepted January 26, 2022; published online February 11, 2022

Citation: Wu, D., Zhou, K., Xiao, M., Liu, Z., and Zhang, S. (2022). Differences in intestinal microbiome are associated with the mortality of COVID-19 patients in intensive care units. Sci China Life Sci 65, 1040-1042. https://doi.org/10.1007/s11427-021-2072-4

Dear Editor,

Coronavirus disease 2019 (COVID-19), caused by infection with severe acute respiratory syndrome coronavirus 2 (SARS-CoV-2), has developed into a global pandemic. In addition to the common respiratory symptoms, a significant proportion of COVID-19 patients experience gastrointestinal symptoms, such as diarrhea, nausea, and vomiting (Parasa et al., 2020). Furthermore, SARS-CoV-2 nucleic acid could be detected in stool samples from COVID-19 patients (Jeong et al., 2020). It is suspected that the gastrointestinal tract might be a site of viral replication and activity. It has been shown that the diversity of the microbiome is significantly reduced in the stools of COVID-19 patients and the taxonomy of the microbiome is also altered significantly ( $\mathrm{Gu}$ et al., 2020). However, it is unknown whether changes in the composition of the intestinal microflora may be associated with the outcome (survival or death) of critically ill COVID-19 patients.

To investigate whether different microbial compositions

$\dagger$ Contributed equally to this work

*Corresponding authors (Zhihua Liu, email: zhihualiu@mail.tsinghua.edu.cn; Shuyang Zhang, email: shuyangzhang103@163.com) may associate with disease outcomes, we carried out a crosssectional pilot study on the relationship between intestinal microbiota and the outcome of critically ill COVID-19 patients. Microbiome samples were analyzed on 21 patients critically ill with confirmed SARS-CoV-2 infection. The 21 patients were admitted to an intensive care unit (ICU) due to critical medical conditions. We also included 11 patients admitted to ICU with severe acute pancreatitis (SAP) as ICU controls. All of the SAP patients survived in our study. Intestinal microbial samples, either stool samples or rectal swabs depending on the availability, were collected within the first $24 \mathrm{~h}$ of their initial admission to ICU. The 21 COVID-19 patients were divided into two groups based on the eventual outcome, survivor $(n=11)$ and non-survivor $(n=10)$ (Figure 1A). Information on recruited patients was listed (Table S1 in Supporting Information). Fecal samples $(n=11)$ or rectal swabs $(n=10)$ from COVID-19 patients were analyzed by high-throughput sequencing of the $16 \mathrm{~S}$ ribosomal RNA (rRNA) gene. Principal component analysis (PCA) analysis of the microbiome showed that stool and swab specimens differed significantly at the genus level (analysis of similarities, $R=0.1855, P=0.0220$ ) (Figure 1B), which is 
A

\begin{tabular}{|c|c|c|c|c|c|}
\hline \multicolumn{4}{|c|}{ COVID-19 } & SAP & Healthy control \\
\hline \multicolumn{2}{|c|}{ Fecal } & \multicolumn{2}{|c|}{ Rectal } & Rectal & Rectal \\
\hline $\begin{array}{c}\text { Survivor } \\
(n=8)\end{array}$ & $\begin{array}{c}\text { Non-survivor } \\
(n=3)\end{array}$ & $\begin{array}{c}\text { Survivor } \\
(n=5)\end{array}$ & $\begin{array}{c}\text { Non-survivor } \\
(n=5)\end{array}$ & $\begin{array}{c}\text { All alive } \\
(n=11)\end{array}$ & $\begin{array}{c}\text { All alive } \\
(n=20)\end{array}$ \\
\hline
\end{tabular}

B

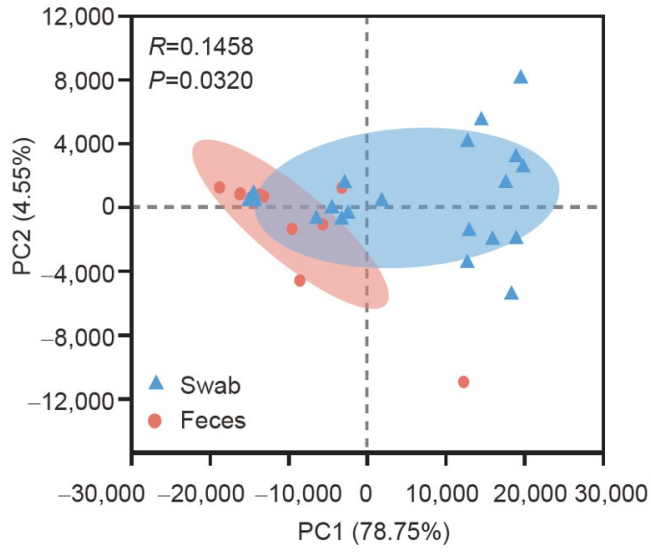

D

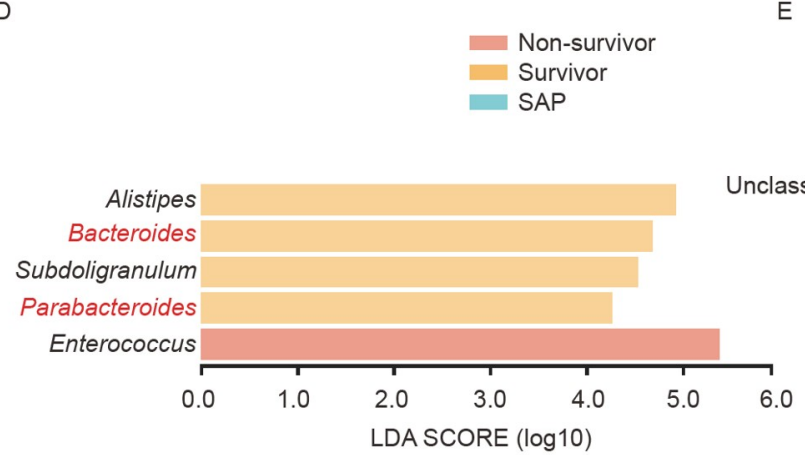

C
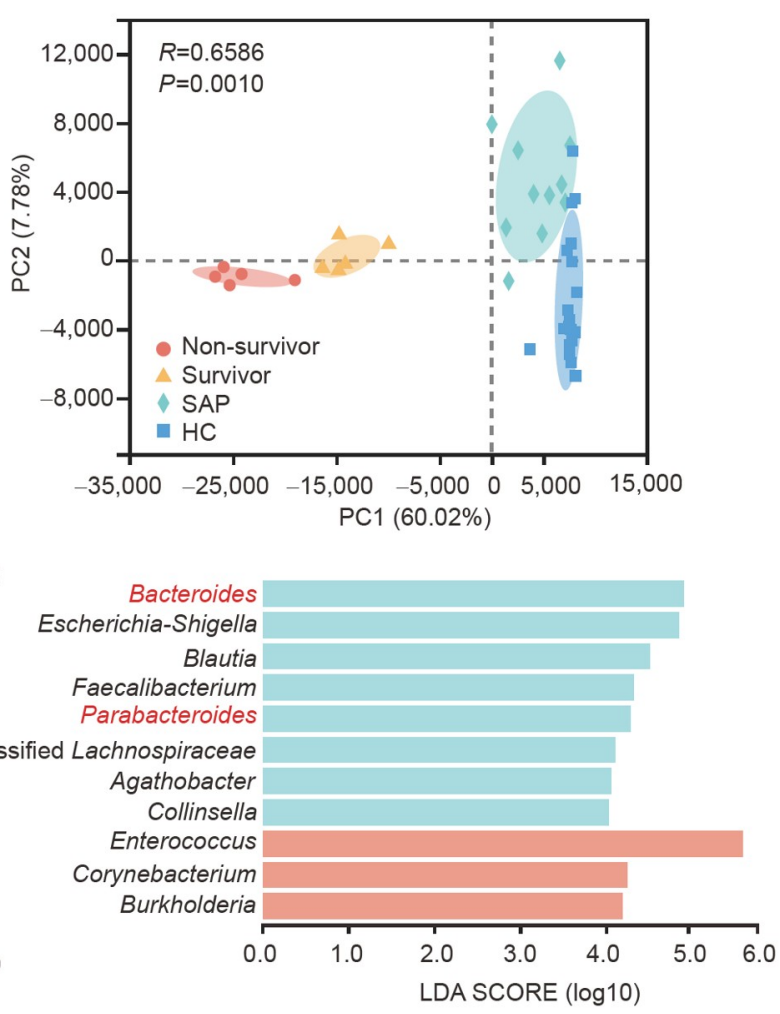

$\mathrm{F}$

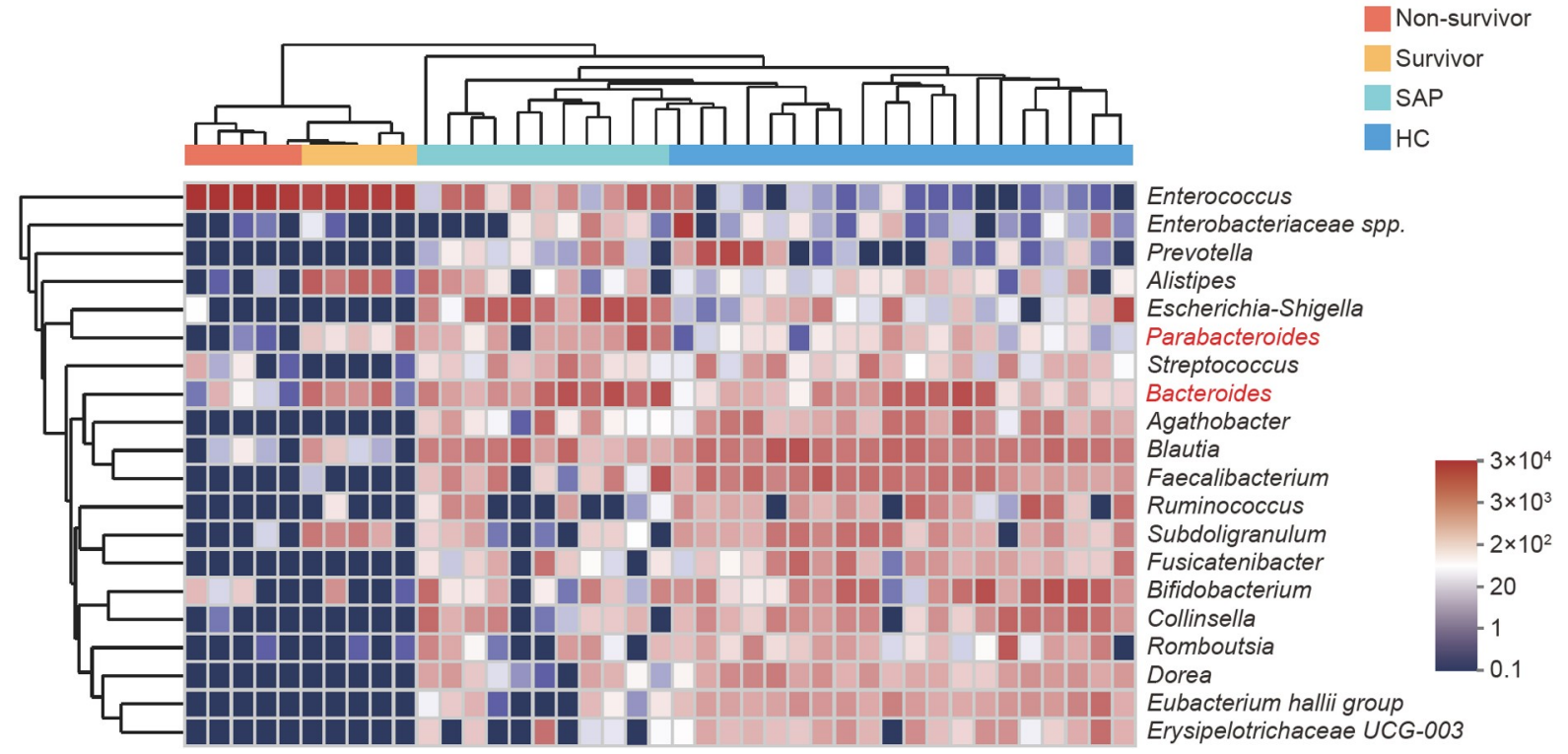

Figure 1 Differences in intestinal microbiome among Survivor, Non-survivor, SAP, and HC. A, Sample subgroups for this study. B, There are significant differences in the $\beta$-diversity of the gut microbiota between the swab and feces groups based on PCA of Bray-Curtis distances. C, There are significant differences in the $\beta$-diversity of the gut microbiota in swab samples among Non-survivor, Survivor, SAP, and HC groups based on PCA of Bray-Curtis distances. D and E, Linear discriminant analysis (LDA) effect size analysis shows significant differences in microbiota composition of swab samples at the genus level between the ICU control and non-survivor patients or survivor patients and non-survivor patients. Only taxa with an LDA threshold $>4.0$ are shown. F, Heat map of the top 20 bacterial genera detected in the individual swab samples. Red, higher abundance; blue, lower abundance. 
consistent with previous reports that microbial compositions are significantly different in mucosa and feces (Carroll et al., 2010; Zoetendal et al., 2002). We performed subsequent analyses on the microbiome from COVID-19 patients by specimen type. PCA analysis at the genus level indicated that the microbiome from swab samples differed between the survivor and non-survivor patients with COVID-19 (Figure 1C). On the contrary, the microbiome from stool samples from COVID-19 did not differ between the survivor group and non-survivor group (Figure S1A-C in Supporting Information). To investigate whether COVID-19 infection might lead to some alternations on microbial composition from other disease conditions, we performed the same analysis on microbiome samples from rectal swabs from patients with SAP or healthy controls (HC). Notably, the microbiome of COVID-19 patients, survival and non-survival group, significantly differed from the microbiome from SAP or HC (Figure 1C), indicating that COVID-19 infection associated with distinct microbial alternations. Two genera, Bacteroides and Parabacteroides, could be selected to distinguish between the survivor and the non-survivor group using linear discriminant analysis effect size (LEfSe) analysis and a random forest model on microbiomes from rectal swab samples (Figure 1D and E; Figure S2A and B in Supporting Information). Finally, unsupervised hierarchical cluster analysis on the top 20 bacterial genera detected in swab samples also indicated a striking difference in bacterial composition between COVID-19 patients and SAP or HC, as well as a difference between the survivor and non-survivor patients with COVID-19 (Figure 1F). Bacteroides and Parabacteroides were among the genera that differed the survivor group from the non-survivor group.

Phenotypically, Bugbase analysis indicated anaerobic bacteria were significantly more abundant in the survivor group, SAP, and HC than those in the non-survivor group (Figure S3A in Supporting Information). To investigate the functional activities of the bacteria, we used the PICRUSt2 tool (Douglas et al., 2020) to map the 16S sequences to the genes and pathways that these bacterial species may harbor. In the survivor group, pyruvate fermentation to propanoate I, mannan degradation, nicotinamide adenine dinucleotide (NAD) biosynthesis I (from aspartate), incomplete reductive tricarboxylic acid (TCA) cycle, and other energy metabolism functions were significantly higher compared to those in the non-survivor group (Figure S3B in Supporting Information).
Overall, our pilot study indicates microbial communities in rectal swabs from critically ill COVID-19 patients were significantly different in taxonomy from SAP ICU patients or healthy control. In addition, the microbial community in rectal swabs differed between the survivors and non-survivors of COVID-19. Microbial energy metabolisms could differ between COVID-19 patients with different disease outcomes. Thus, our pilot study suggests that the intestinal microflora in COVID-19 patients during hospitalization in an ICU was different based on mortality. Nevertheless, the current study is limited because this is a single-center crosssectional study with a very small sample size. It awaits to be validated in regions with sufficient numbers of COVID-19 ICU patients.

Compliance and ethics The author(s) declare that they have no conflict of interest.

Acknowledgements This work was supported by the National Key Research and Development Program of China (2020YFC0861000 and 2018YFE0207300), Beijing Nova Program (Z201100006820127), and the CAMS Endowment Fund (2021-CAMS-JZ004). The study protocol was approved by the Research Ethical Committee of Peking Union Medical College Hospital (Approval No. SK1297).

\section{References}

Carroll, I.M., Chang, Y.H., Park, J., Sartor, R.B., and Ringel, Y. (2010). Luminal and mucosal-associated intestinal microbiota in patients with diarrhea-predominant irritable bowel syndrome. Gut Pathog 2, 1-9.

Douglas, G.M., Maffei, V.J., Zaneveld, J.R., Yurgel, S.N., Brown, J.R., Taylor, C.M., Huttenhower, C., and Langille, M.G.I. (2020). PICRUSt2 for prediction of metagenome functions. Nat Biotechnol 38, 685-688.

Gu, S., Chen, Y., Wu, Z., Chen, Y., Gao, H., Lv, L., Guo, F., Zhang, X., Luo, R., Huang, C., et al. (2020). Alterations of the gut microbiota in patients with coronavirus disease 2019 or H1N1 influenza. Clin Infect Dis 71, 2669-2678.

Jeong, H.W., Kim, S.M., Kim, H.S., Kim, Y.I., Kim, J.H., Cho, J.Y., Kim, S.H., Kang, H., Kim, S.G., Park, S.J., et al. (2020). Viable SARS-CoV-2 in various specimens from COVID-19 patients. Clin Microbiol Infect 26, 1520-1524.

Parasa, S., Desai, M., Thoguluva Chandrasekar, V., Patel, H.K., Kennedy, K.F., Roesch, T., Spadaccini, M., Colombo, M., Gabbiadini, R., Artifon, E.L.A., et al. (2020). Prevalence of gastrointestinal symptoms and fecal viral shedding in patients with coronavirus disease 2019. JAMA Netw Open 3, e2011335.

Zoetendal, E.G., von Wright, A., Vilpponen-Salmela, T., Ben-Amor, K., Akkermans, A.D.L., and de Vos, W.M. (2002). Mucosa-associated bacteria in the human gastrointestinal tract are uniformly distributed along the colon and differ from the community recovered from feces. Appl Environ Microbiol 68, 3401-3407.

\section{SUPPORTING INFORMATION}

The supporting information is available online at https://doi.org/10.1007/s11427-021-2072-4. The supporting materials are published as submitted, without typesetting or editing. The responsibility for scientific accuracy and content remains entirely with the authors. 